\title{
Magnetic field effect and dielectric anomalies at the spin reorientation phase transition of $\mathrm{GdFe}_{3}\left(\mathrm{BO}_{3}\right)_{4}$
}

\author{
F. Yen, ${ }^{1}$ B. Lorenz, ${ }^{1}$ Y. Y. Sun, ${ }^{1}$ C. W. Chu,${ }^{1,2,3}$ L. N. Bezmaternykh,${ }^{4}$ and A. N. Vasiliev ${ }^{5}$ \\ ${ }^{1}$ Texas Center for Superconductivity of the University of Houston \\ and Department of Physics, University of Houston, \\ Houston, Texas 77204-5002, USA \\ ${ }^{2}$ Lawrence Berkeley National Laboratory, 1 Cyclotron Road, Berkeley, California 94720, USA \\ ${ }^{3}$ Hong Kong University of Science and Technology, Hong Kong, China \\ ${ }^{4}$ Institute of Physics, Siberian Division, Russian Academy of Sciences, Krasnoyarsk, 660036 Russia \\ ${ }^{5}$ Faculty of Physics, Moscow State University, Moscow, 119992 Russia
}

(Received 9 August 2005; published 27 February 2006)

\begin{abstract}
$\mathrm{GdFe}_{3}\left(\mathrm{BO}_{3}\right)_{4}$ exhibits a structural phase transition at $156 \mathrm{~K}$, antiferromagnetic order of the $\mathrm{Fe}^{3+}$ moments at $36 \mathrm{~K}$, followed by a spin reorientation phase transition at $9 \mathrm{~K}$. The reorientation phase transition is studied through dielectric, magnetic, and heat capacity measurements under the application of external magnetic fields of up to $7 \mathrm{kOe}$. The dielectric constant indicates the existence of two distinct anomalies at $T_{S R}=9 \mathrm{~K}$ that separate in temperature under external magnetic fields. The spin rotation phase transition is proven to be of the first-order nature through the magnetic analog of the Clausius-Clapeyron equation. Magnetodielectric effect of up to $1 \%$ is observed at $8 \mathrm{~K}$ and $7 \mathrm{kOe}$. The uniaxial magnetocaloric effect along the $c$ axis is observed below the spin reorientation phase transition of $9 \mathrm{~K}$.
\end{abstract}

DOI: 10.1103/PhysRevB.73.054435

PACS number(s): 75.30.Kz, 75.50.Ee, 75.80.+q, 77.80.-e

$\mathrm{GdFe}_{3}\left(\mathrm{BO}_{3}\right)_{4}$ belongs to the trigonal system with space group $R 32$. It is similar to huntite $\mathrm{CaMg}_{3}\left(\mathrm{CO}_{3}\right)_{4}$, a trigonal trapezohedral structure that is one of the five trigonal types. Borate crystals of this category are appealing because of their possible applications as single crystal minilasers due to their good luminescent and nonlinear optical properties. In particular for $\mathrm{GdFe}_{3}\left(\mathrm{BO}_{3}\right)_{4}$ single crystals, recent studies have focused on the better understanding of its optical properties, and at the same time on its magnetic properties through phase matching of absorption and second harmonic generation spectra. ${ }^{1,2}$ The magnetic properties of the rareearth iron borates are also of fundamental interest because of the existence and mutual interference of two magnetic subsystems ( $\mathrm{Fe}$ and $\mathrm{Gd}$ ). The understanding of the magnetic orders of the gadolinium and iron sublattices, and the coupling between the iron spins and the gadolinium moments that contributes to the crystal's rich magnetic properties is of fundamental importance.

The crystal structure of huntite has been analyzed and described elsewhere, ${ }^{3-5}$ it consists of $\mathrm{GdO}_{6}$ bipyramids and $\mathrm{FeO}_{6}$ octahedrons. The octahedrons form threefold helicoidal chains along the $c$ axis. The bipyramids are located between three nearly equal distant octahedrons. Rare-earth iron borates, $R \mathrm{Fe}_{3}\left(\mathrm{BO}_{3}\right)_{4}(R=\mathrm{Eu}$ to $\mathrm{Ho}$, and $\mathrm{Y})$, undergo a structural transition at higher temperatures and an antiferromagnetic (AFM) transition involving the Fe spins at $T_{N} \approx 35 \mathrm{~K}^{6}{ }^{6}$ For $R=\mathrm{Gd}$ a weakly first-order structural phase transition at $T_{1}=156 \mathrm{~K}$ changes the structural symmetry from $R 32$ to $P 3{ }_{1} 2_{1}$. ${ }^{5}$ A second-order phase transition at $T_{N}=36 \mathrm{~K}$ results in the AFM ordering of the $\mathrm{Fe}^{3+}$ magnetic moments aligned in the basal plane. At $T_{S R}=9 \mathrm{~K}$, another sharp phase transition occurs characterized by a spin reorientation of the $\mathrm{Fe}^{3+}$ magnetic moments by $90^{\circ}$ from the basal plane to the $c$ axis.

The coupling between the $\mathrm{Gd}$ moments and the Fe spins at low temperatures is strong in $\mathrm{GdFe}_{3}\left(\mathrm{BO}_{3}\right)_{4}$ and it was speculated that the reorientation of the AFM iron spin system is triggered by the anisotropy of the Gd moments aligned with the $c$ axis. ${ }^{6}$ This exchange interaction is indirect and involves other ions such as oxygen in the structure. Below $T_{N}$, with decreasing temperature, the magnetic order grows, and that causes strain via the spin-lattice interaction. At the spin reorientation phase transition the magnetic order experiences a sudden change that is reflected in distinct anomalies of the dielectric constant, $\epsilon$, as recently discussed in several rare-earth manganites. ${ }^{7,8}$ The dielectric constant can be measured with extraordinary precision and it monitors subtle changes of the magnetic system. We have therefore searched for dielectric anomalies at the magnetic phase transitions in $\mathrm{GdFe}_{3}\left(\mathrm{BO}_{3}\right)_{4}$ and its correlation with magnetic and heat capacity data. Below $T_{N}$, close to $T_{S R}$, we found two distinct anomalies of $\epsilon$ that separate in temperature with applied magnetic fields. A sharp drop of $\epsilon$ occurs at the reorientation of the Fe spins, whereas a maximum of $\epsilon(T)$ above $T_{S R}$ indicates the onset of magnetic-field-induced ferroelectric order. The spin reorientation phase transition is proven to be of the first-order nature through the magnetic ClausiusClapeyron equation. Lastly, a phase diagram is proposed for magnetic fields parallel or perpendicular to the crystalline $c$ axis.

The single crystal $\mathrm{GdFe}_{3}\left(\mathrm{BO}_{3}\right)_{4}$ was grown as described elsewhere. ${ }^{9}$ The transparent crystal (shining green in color) was analyzed and oriented using a GADDS x-ray diffractometer. The heat capacity data was acquired via Quantum Design's physical property measurement system (PPMS) under the application of magnetic fields of up to $6 \mathrm{kOe}$ both along the $a$ and $c$ axes (we use the hexagonal coordinate system in which $a \perp c$ ). Direct current magnetization data was measured employing Quantum Design's magnetic prop- 


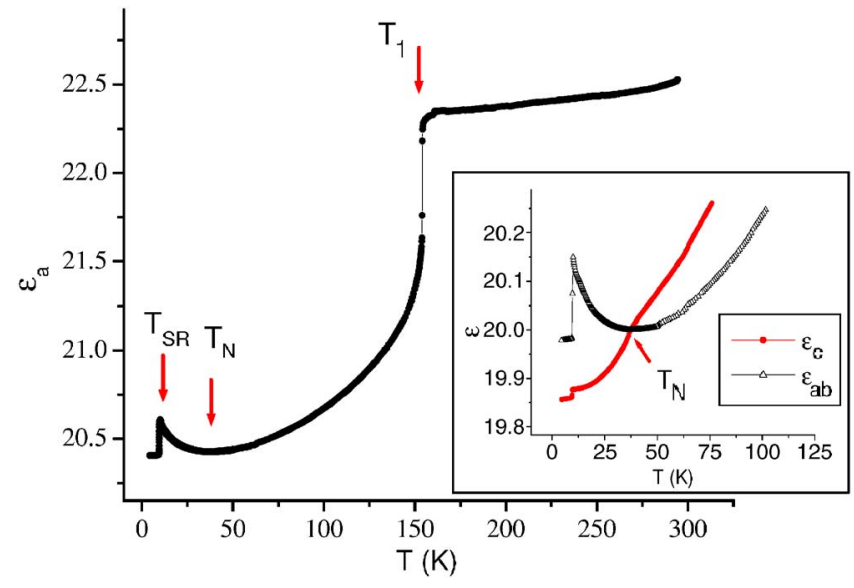

FIG. 1. (Color online) Dielectric data showing all three major anomalies. $T_{1}=156 \mathrm{~K}, T_{N}=36 \mathrm{~K}$, and $T_{S R}=9 \mathrm{~K}$. The inset shows the behavior of $\epsilon(T)$ around $T_{N}$ when $\epsilon$ is measured along the hexagonal $a$ and $c$ axis for $H=0$.

erty measurement system (MPMS) in magnetic fields parallel to $a$ and $c$. Smaller portions of the crystal were cut from an original crystal in order to align the two desired crystallographic orientations for dielectric investigations. Silver paint was used as electrodes for the dielectric measurements and the sample was mounted onto a homemade capacitance probe that was adapted to the PPMS. The capacitance was measured by the high precision capacitance bridge AH2500A (Andeen Hagerling) operating at a frequency of $1 \mathrm{kHz}$. The temperature and magnetic fields were controlled by the PPMS when the dielectric constant was measured.

The three known phase transitions are reflected in different anomalies of the dielectric constant (Fig. 1). At $T_{1}$ $=156 \mathrm{~K}$, the dielectric constant drops significantly since the transition is a structural one. $T_{1}$ does not depend on magnetic field. The magnetic transition into the AFM2 phase at $T_{N}$ is distinguished by a change in slope of $\epsilon_{c}$ and a minimum of $\epsilon_{a}$ at $36 \mathrm{~K}$. Below $T_{N}, \epsilon_{a}$ starts increasing with decreasing $T$ and develops the peaklike feature at $T_{S R}$, the transition into the AFM1 phase, as shown in Fig. 1. This is a clear signature of lattice softening and it has to be correlated with the changes in the magnetic order at and below $T_{N}$. A similar, but positive slope change at $T_{N}$ is observed in $\epsilon_{c}$. This behavior is analogous to the magnetic data that will be discussed later. In a system where there are two magnetic subsystems present with one of them being a $d$-metal and the other one an $f$-metal subsystem, the $f$ metal is magnetically polarized upon the ordering of the $d$-metal subsystem. ${ }^{10}$ In $\mathrm{GdFe}_{3}\left(\mathrm{BO}_{3}\right)_{4}$ the gadolinium develops an AFM order along the $c$ axis through interplanar exchange interactions mediated by the Gd-O-Fe bonds below $T_{N}$. The dielectric anomalies observed at the magnetic phase transitions are an indication of strong spin-lattice interactions and are attributed to magnetoelastic effects similar to those observed in some rareearth manganites; ${ }^{11}$ a strong coupling of the dielectric response with the rare-earth magnetic moment was also reported in these compounds. ${ }^{7,8}$ The softness of the lattice [as expressed by the sharp increase of $\left.\epsilon_{a}(T)\right]$ is therefore an indication of the softening of the magnetic order as $T$ ap-

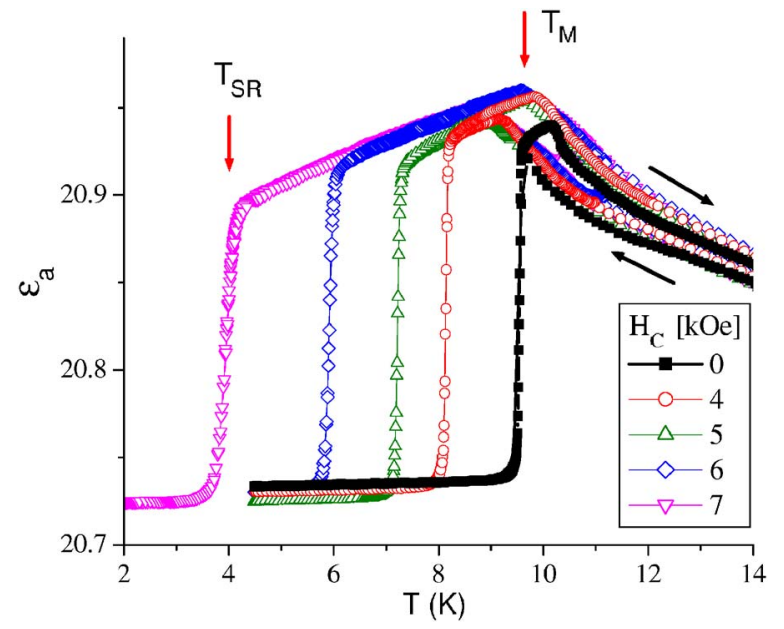

FIG. 2. (Color online) Splitting of the spin reorientation phase transition under fields parallel to the $c$ axis. $T_{S R}$ shifts lower while $T_{M}$ stays the same.

proaches $T_{S R}$, resulting in the Fe-spin reorientation triggered by the Gd moments and the abrupt decrease of $\epsilon_{a}$. Thermal hysteresis was observed in $\epsilon(T)$ above $T_{S R}$ at zero magnetic field. The hysteretic behavior of $\epsilon_{a}(T)$ extends over several degrees just above $T_{S R}$. Upon cooling in zero field the dielectric constant below $T_{N}$ increases continuously for $T \rightarrow T_{S R}$ and drops rapidly right below $T_{S R}$. However, upon heating, after the sharp increase at $T_{S R}$ there appears a distinct maximum of $\epsilon_{a}$ at $T_{M}$ about $0.7 \mathrm{~K}$ higher in temperature (Fig. 2). This second anomaly at $T_{M}$ needs to be explored in more detail.

The external magnetic field, $H$, shows an interesting effect on both dielectric anomalies $\left(T_{S R}\right.$ and $\left.T_{M}\right)$. If $H$ is aligned with the $c$ axis, the spin reorientation transition is suppressed and $T_{S R}$ quickly decreases with $H_{c}$ in accordance with recent magnetic data. ${ }^{9}$ However, $T_{M}$ is barely affected by $H_{c}$, and the separation of both anomalies increases in magnetic fields as shown in Fig. 2. In contrast, for magnetic fields parallel to $a, T_{S R}$ remains constant but $T_{M}$ shifts to higher temperature (Fig. 3). At the same time, the enhancement of $\epsilon_{a}(T)$ is largely reduced and disappears for fields exceeding $7 \mathrm{kOe}$. This qualitative behavior of the dielectric constant in magnetic fields is closely correlated with the magnetic properties of the coupled system of the Fe spins and the Gd moments. It is interesting to note that recent measurements of the magnetic field induced electric polarization, $P$, of $\mathrm{GdFe}_{3}\left(\mathrm{BO}_{3}\right)_{4}$ have shown a sudden increase of $P$ in crossing $T_{S R}$ with increasing field. ${ }^{12}$ This may indicate the existence of a fieldinduced ferroelectric phase (FIP) and it was proposed that higher-order magnetoelectric couplings are responsible for the observed phenomena. From our dielectric measurements we can uniquely identify the boundaries of this new phase by the sudden drop of $\epsilon_{a}(T)$ at $T_{S R}$ and the distinct maximum of $\epsilon_{a}(T)$ at the high temperature end $\left(T_{M}\right)$. Therefore the phase diagram of $\mathrm{GdFe}_{3}\left(\mathrm{BO}_{3}\right)_{4}$ includes three distinct phases (AFM2, AFM1, and FIP) below the AFM ordering temperature, $T_{N}$, of the iron system.

The results of magnetization measurements with fields oriented parallel to $a$ and $c$ are summarized in Fig. 4. Sur- 


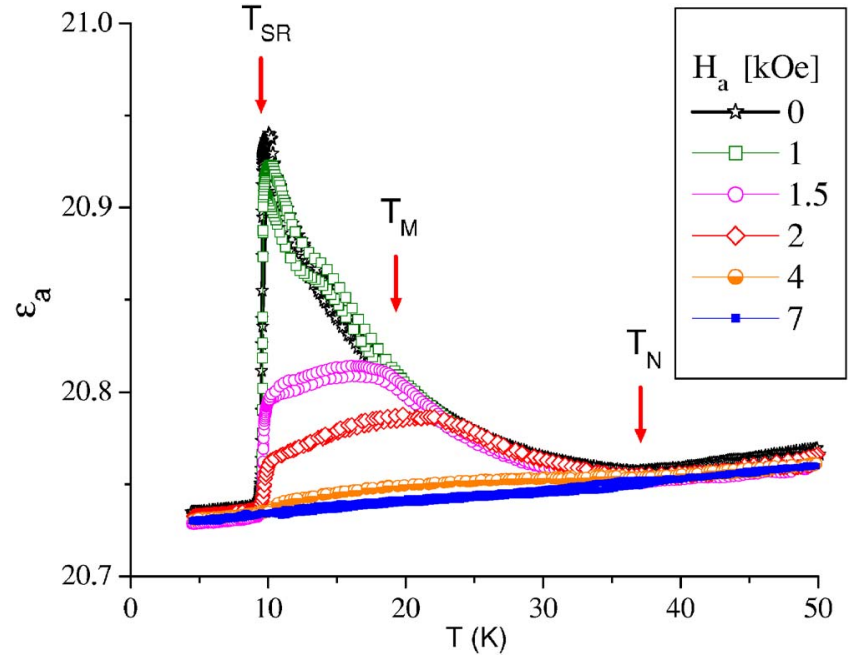

FIG. 3. (Color online) Splitting of the spin reorientation phase transition under fields parallel to the $a$ axis. $T_{S R}$ remains constant while $T_{M}$ shifts higher.

prisingly, the $a$ - and $c$-axes dc susceptibilities are equal above $T_{N}$ within the experimental resolution, i.e., $\mathrm{GdFe}_{3}\left(\mathrm{BO}_{3}\right)_{4}$ is magnetically isotropic at high temperatures although there is evidence for easy plane anisotropy of the Fe spins and easy axis anisotropy of the Gd spins. ${ }^{6}$ The anisotropies of both magnetic ions are obviously correlated resulting in a complete isotropic balancing just like some rare-earth metal ferrite-garnets and orthoferrites. ${ }^{13}$ The high temperature Curie-Weiss extrapolation yields a Curie-Weiss temperature of $\Theta_{C W}=-34 \mathrm{~K}$, and an effective magnetic moment $\mu_{e f f}=12.2 \mu_{B}$ in good agreement with other reports ${ }^{14}$ and the theoretically expected value of $\mu_{e f f}=12.96 \mu_{B}\left(\mathrm{Gd}^{3+}\right.$, $S=7 / 2$ and $\mathrm{Fe}^{3+}, S=5 / 2$ ). Deviations from the Curie-Weiss behavior due to short-range AFM correlations are detected below $100 \mathrm{~K}$.

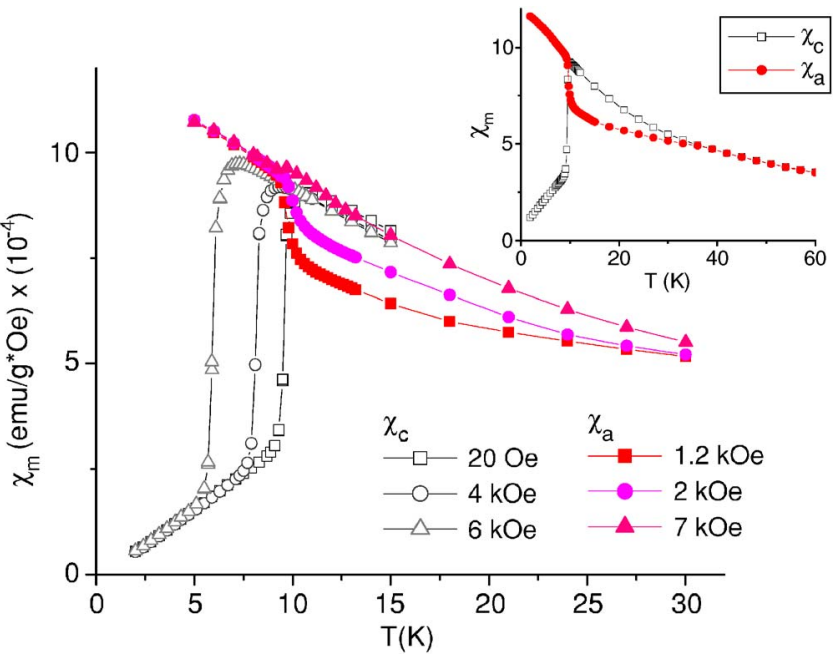

FIG. 4. (Color online) Mass susceptibility data at different magnetic fields parallel and perpendicular to the $c$ axis. $T_{S R}$ shifts lower and $\Delta M$ increases under magnetic fields parallel to the $c$ axis (open). $T_{S R}$ stays the same if magnetic fields are aligned with the $a$ axis (solid). $\chi_{a}$ is only affected between $T_{S R}<T<T_{N}$.
Below $T_{N}$ the magnetic response becomes anisotropic and depends on the direction of the probing field. For $H \| c$, the dc susceptibility, $\chi_{c}(T)$, does not exhibit any anomaly at $T_{N}$ and it is independent of the magnetic field (up to $10 \mathrm{kOe}$ ) between $T_{N}$ and $T_{S R} \cdot \chi_{c}$ decreases suddenly at $T_{S R}$ as a result of the spin reorientation aligning the Fe spins with the $c$ axis (Fig. 4). The major effect of $H_{c}$ is the shift of $T_{S R}$ to lower $T$ and eventually the suppression of the spin reorientation for fields above $8 \mathrm{kOe}$. It is obvious that the magnetic response strongly depends on the angle between the external field and the Fe spins. This is justifiable since it is energetically unfavorable to keep the Fe spins aligned with the external magnetic field. This qualitative behavior is reflected in the field dependence of the dielectric constant (Fig. 2). $\epsilon_{a}(T)$ increases below $T_{N}$ and exhibits a distinct maximum at $T_{M} \approx 10 \mathrm{~K}$. The sharp drop of $\epsilon_{a}(T)$ at lower temperature coincides with $T_{S R}(H)$ as determined magnetically. $\epsilon_{a}(T)$ is only weakly dependent on the field $H_{c}$ between $T_{N}$ and $T_{M}$ as is the magnetic susceptibility, $\chi_{c}$. Whereas the value of $T_{M}$ is not affected by the field oriented along the $c$ axis $T_{S R}(H)$ decreases rapidly reaching zero at about $8 \mathrm{kOe}$ and separating the two dielectric anomalies in the magnetic field. The peak of $\epsilon_{a}(T)$ indicating the phase boundary between the AFM2 phase and the FIP phase exists well above $8 \mathrm{kOe}$, the critical field that suppresses the AFM1 phase. Details of the phase diagram will be discussed later.

The magnetic response to an in-plane magnetic field, $H_{a}$, is completely different. At low magnetic fields, the susceptibility $\chi_{a}$ levels off below $T_{N}$ and is clearly lower than $\chi_{c}$ (Fig. 4). This can be attributed to the AFM order of the Fe spins below $T_{N}$, reducing the susceptibility for $H \| a$. Close to $T_{S R}$, however, $\chi_{a}$ increases again and its temperature dependence below $T_{S R}$ appears to be a continuation of the $c$-axis susceptibility, $\chi_{c}$ (discussed above). This behavior agrees well with the observation that the magnetic susceptibility is little affected by the AFM order if the field is perpendicular to the Fe spins. At $T_{S R}$ the Fe spins rotate towards the $c$ axis and align themselves with the magnetic field. $\chi_{a}(H)$ rapidly increases with $H$ in the temperature range $T_{S R}<T<T_{N}$, and it approaches the values of $\chi_{c}$ above $7 \mathrm{kOe} . T_{S R}$ is independent of $H_{a}$, as is also reflected in the dielectric data of Fig. 3 [the sharp drop of $\epsilon_{a}(T)$ marks the spin reorientation transition]. $\epsilon_{a}(T)$ between $T_{S R}$ and $T_{N}$, however, is strongly affected by $H_{a}$, and its enhancement below $T_{N}$ is reduced by the magnetic field. Thereby the maximum of $\epsilon_{a}(T)$ is shifted to higher temperatures (Fig. 3) reaching the Néel temperature at a relatively low field of 4 to $5 \mathrm{kOe}$. This limits the AFM2 phase and extends the temperature range of the FIP phase to $T_{N}=36 \mathrm{~K}$.

The dielectric anomalies observed in $\mathrm{GdFe}_{3}\left(\mathrm{BO}_{3}\right)_{4}$ at the magnetic transitions reveal an interesting correlation between the different magnetic subsystems (Fe and Gd) and the spin-lattice coupling leading to the modifications of $\epsilon(T)$. To arrive at a deeper understanding the magnetic correlations have to be considered in more detail. The phase diagram and the magnetic order of $\mathrm{GdFe}_{3}\left(\mathrm{BO}_{3}\right)_{4}$ was recently investigated by AFM resonance experiments and it was suggested that the Fe spins at high temperatures are aligned in the $a-b$ plane (easy plane anisotropy) whereas the Gd moment experience a 
strong easy axis anisotropy orienting them along the $c$ axis. ${ }^{6}$ The AFM order of the iron moments below $T_{N}$ couples to the gadolinium moments resulting in an AFM alignment of the Gd spins ("magnetic polarization"). With decreasing temperature the Fe-Gd coupling grows stronger and eventually triggers the reorientation of the $\mathrm{Fe}$ spins resulting in a transition from the noncollinear $\left(T_{S R}<T<T_{N}\right)$ to a collinear $\left(T<T_{S R}\right)$ magnetic order of both subsystems along the $c$ axis. This phenomenon, related to the strong single ion anisotropy of rare-earth ions, is not uncommon and it was observed in a number of different compounds involving rareearth ions and $d$ elements. ${ }^{15-17}$ Depending on the strength of the anisotropy factors, the coupling between the $f$ moments and $d$ spins results in the rotation of the $d$ spins towards the $f$ moments (such as in $R_{2} \mathrm{CuO}_{4}, R=\mathrm{Pr}, \mathrm{Nd}, \mathrm{Sm}, \mathrm{Eu}$ ) ${ }^{15,18}$ or vice versa (e.g., in orthorhombic $\mathrm{HoMnO}_{3}$ ). ${ }^{16}$ In rare cases the $f$ - $d$ exchange can result in an in-plane rotation of the $d$ spins as observed in hexagonal $\mathrm{HoMnO}_{3} .{ }^{19}$ Dielectric anomalies associated with spin reorientation transitions have been observed in several manganites, ${ }^{7,20}$ and the significance of the spin-lattice coupling and magnetoelastic effects has been shown. ${ }^{11}$ The coupling between the $f$ and $d$ spins forming a $90^{\circ}$ angle can be mediated by the antisymmetric Dzyaloshinskii-Moriya interaction (which is proportional to the vector product of $f$ and $d$ moments and gives rise to weak ferromagnetism), or by a pseudodipolar interaction arising from the anisotropy of the $f-d$ exchange. ${ }^{14}$ In any case, the exchange interaction maximizes if the two moments are perpendicular to each other.

The Fe spin reorientation observed in $\mathrm{GdFe}_{3}\left(\mathrm{BO}_{3}\right)_{4}$ at $T_{S R}$ is triggered by this $f-d$ exchange mechanism. We propose that the observed enhancement of the dielectric constant below $T_{N}$ (Figs. 1 to 3 ) is closely related to the indirect $f-d$ exchange coupling and the magnetic polarization of the Gd moments. The increase of $\epsilon_{a}(T)$ as $T$ approaches $T_{S R}$ at $H=0$ reflects the increased coupling strength and AFM Gd sublattice polarization. The spin-phonon coupling causes the lattice to soften as the magnetic order becomes unstable for $T \rightarrow T_{S R}$. The combined effects on $\epsilon_{a}$ saturate at $T_{S R}(H=0)$ and it disappears abruptly with the spin reorientation of the iron at lower $T$ resulting in the sudden decrease of the dielectric constant. With the magnetic field applied along the $c$ axis $T_{S R}$ is lowered but the polarizing effect on the Gd moments and the enhancement of $\epsilon_{a}(T)$ still reaches its maximum at $9 \mathrm{~K}$ explaining the peak of $\epsilon_{a}$ remaining $H$ independent at $T_{M} \approx 9 \mathrm{~K}$. With the magnetic field applied along the $a$ axis, the Gd moments are tilted away from the $c$ axis and the in-plane AFM order of the Fe spins is gradually reduced, as can be concluded from the field effect on the susceptibility, $\chi_{a}$ (Fig. 4). Both effects reduce the $f-d$ exchange coupling, and the magnetic polarization of the Gd-spins and its effect on the lattice is reduced. The saturation of the lattice softness happens at higher temperature in the in-plane magnetic field, the maximum of $\epsilon_{a}(T)$ is suppressed, and $T_{M}$ increases with $H$ (Fig. 3). Although this discussion is qualitative it does explain the two anomalies observed in the temperature dependence of the dielectric constant and their opposite dependence on the magnetic fields parallel or perpendicular to the $c$-axis. The softening of the lattice at $T_{M}$ eventually results in

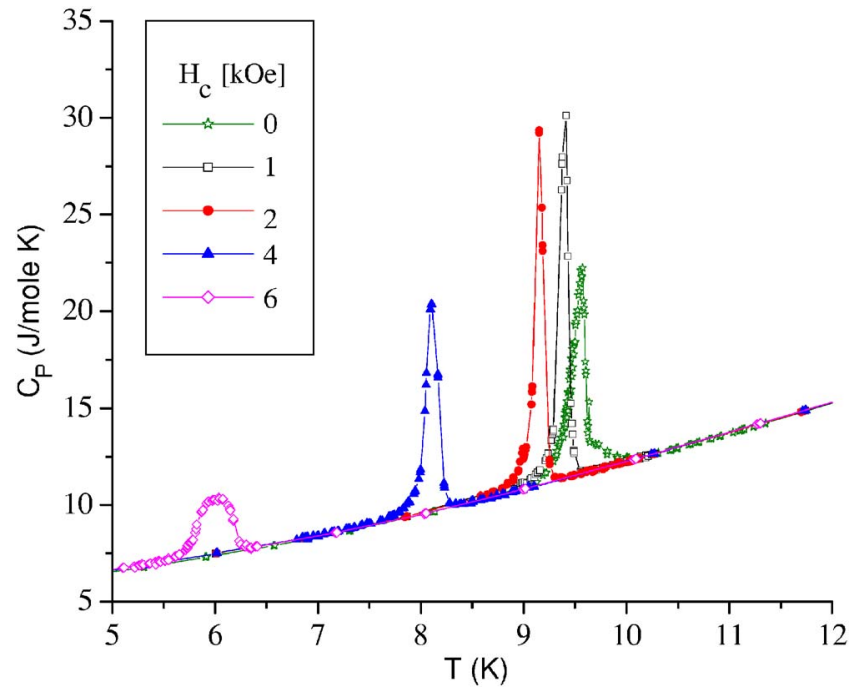

FIG. 5. (Color online) Heat capacity data at different magnetic fields parallel to the $c$ axis. $T_{S R}$ shifts lower and $\Delta S$ decreases with $H_{c}$.

a ferroelectric displacement as observed in recent polarization measurements ${ }^{12}$ that indicate the existence of a sizable field-induced polarization between $T_{S R}$ and $T_{M}$ for both orientations ( $c$ axis and in-plane) of the magnetic field. The corresponding magnetostriction data provide the experimental proof for the lattice anomalies at the phase boundaries of the FIP phase, $T_{S R}$ and $T_{M}$.

The thermodynamic signature of the magnetic phase transitions at $T_{N}$ and $T_{S R}$ is given by sharp peaks of the zero-field heat capacity ${ }^{21}$ at the phase transitions. In external magnetic fields, $H \| c$, the heat capacity peak at $T_{S R}$ shifts to lower $T$, in accordance with the magnetic and dielectric data discussed above. Figure 5 shows the heat capacity close to $T_{S R}$ at different values of $H_{c}$. All peaks are relatively sharp and symmetrical indicative of a first-order phase transition. The heat capacity peak does not shift with $H \| a$, which is consistent with our dielectric and magnetic data. From the structural point of view the exchange interaction between gadolinium ions should be weak since they are interconnected by $\mathrm{BO}_{3}$ triangles that are $2.4 \AA$ apart. Thus $T_{M}$ is not observed in the heat capacity or magnetization data but only in dielectric data.

The magnetic phase diagram of $\mathrm{GdFe}_{3}\left(\mathrm{BO}_{3}\right)_{4}$ at lower temperatures derived from the heat capacity, magnetization, and dielectric measurements is shown in Fig. 6 for both orientations of the magnetic field. $T_{S R}$ is not affected by $H_{a}$, however; it decreases quadratically with $H_{c}$, in agreement with the results of recent resonance ${ }^{6}$ and magnetoelectric experiments. ${ }^{12}$ The field dependence of $T_{M}$ is also shown in Fig. 6. $T_{M}$ merges with $T_{N}$ for $H_{a} \approx 5 \mathrm{kOe}$ in good agreement with the anomalies detected in polarization and magnetostriction measurements. However, for $H \|_{c}, T_{M}(H)$ is almost constant and extends to far higher fields than $T_{S R} . T_{S R}$ and $T_{M}$ form the phase boundaries of the FIP phase where magnetic field-induced polarization was observed. ${ }^{12}$ It is interesting to note that the heat capacity does not show any anomaly at $T_{M}$ as it does at $T_{S R}$. The transition across $T_{M}$ is obviously not 

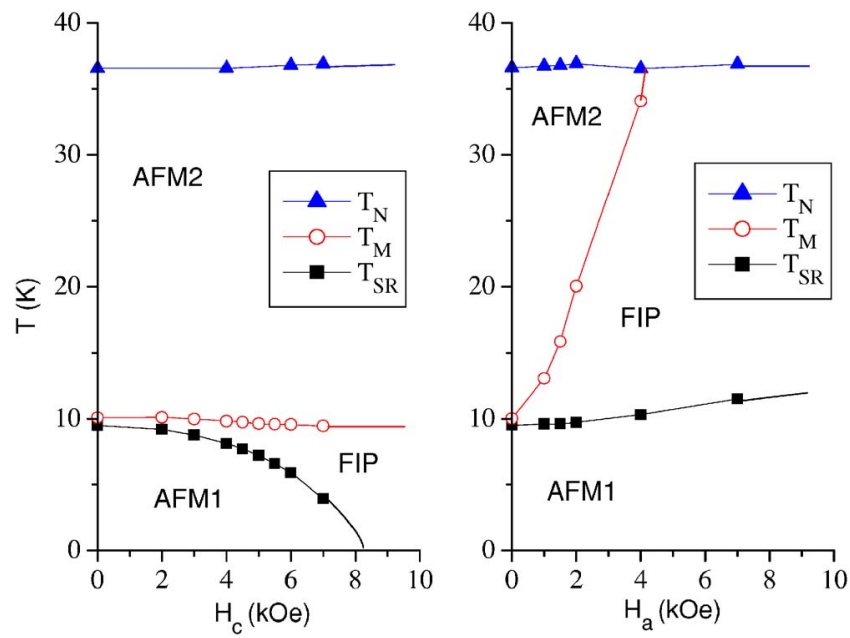

FIG. 6. (Color online) Phase diagram of $\mathrm{GdFe}_{3}\left(\mathrm{BO}_{3}\right)_{4}$ at low temperatures including $T_{S R}, T_{M}$, and $T_{N}$ derived from dielectric data. AFM1 is the state where the Fe spins are ordered antiferromagnetically along the basal plane, AFM2 is the state where the Fe spins are ordered antiferromagnetically along the $c$ direction. FIP is the state where there is field-induced polarization.

accompanied by anomalies such as a sizable change of volume, etc. This indicates a more subtle change in the correlated magnetic systems of $\mathrm{Gd}$ and $\mathrm{Fe}$ spins coupled to the lattice that results in the distinct peak of the dielectric constant.

Given the sharp nature of the heat capacity peak anomaly at $T_{S R}$ as well as its sudden drop in magnetization and dielectric constant, a first-order phase transition is presumed to occur. To further verify the validity of the first-order nature at $T_{S R}$ we make use of the magnetic analog of the ClausiusClapeyron equation,

$$
-\Delta S(B) / \Delta M(B)=d B / d T_{S R}(B),
$$

where $\Delta S$ is the change in entropy, $\Delta M$ is the change in magnetization, and $d B / d T_{S R}$ is the inverse slope of $T_{S R}(B)$ with respect to the internal magnetic field $B$. The set of values for $\Delta S$ are obtained from integrating the area underneath the peak anomaly of $C p / T$ at different fields (Fig. 5). The set of values for $\Delta M$ is obtained from the change of the magne-

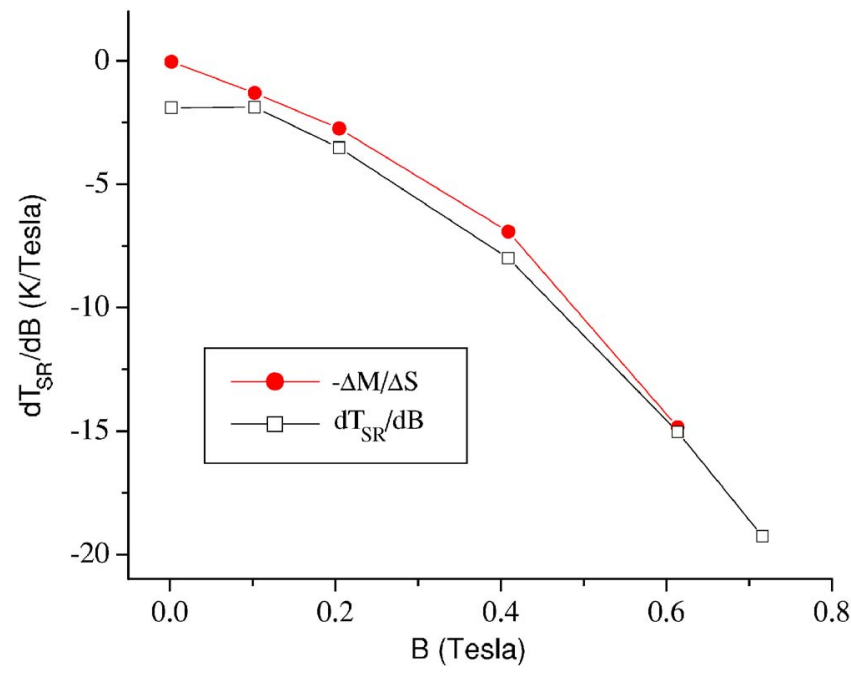

FIG. 7. (Color online) The Clausius-Clapeyron equation satisfied at different magnetic fields $H_{c}$.

tization at $T_{S R}$ (Fig. 4). $d B / d T_{S R}$ is extracted from the phase diagram of Fig. 6 and $B=\mu_{0}(H+M)$. For the case of $H \| c$, Eq. (1) is well fulfilled as shown in Fig. 7. This proves the first-order nature of the spin reorientation transition.

In conclusion, we have demonstrated a strong correlation between the magnetic order and the dielectric properties of $\mathrm{GdFe}_{3}\left(\mathrm{BO}_{3}\right)_{4}$. Below the AFM ordering temperature of the Fe spins, $T_{N}, \epsilon_{a}(T)$ increases and reaches a maximum at the spin reorientation transition temperature, $T_{S R}$, in zero magnetic field. We interpret the apparent lattice softness as a consequence of the indirect exchange coupling between the $\mathrm{Gd}$ and $\mathrm{Fe}$ spins. For nonzero magnetic fields the $\epsilon_{a}(T)$ maximum (at $\left.T_{M}\right)$ and the spin reorientation transition are separated and appear at different temperatures. The spin reorientation transition is proven to be a first-order phase transition through the magnetic analog of the Clausius-Clapeyron equation.

This work is supported in part by NSF Grant No. DMR9804325, the T.L.L. Temple Foundation, the J. J. and R. Moores Endowment, and the State of Texas through the TCSUH and at LBNL by the DoE.
${ }^{1}$ A. M. Kalashnikova, V. V. Pavlov, R. V. Pisarev, L. N. Bezmaternykh, M. Bayer, and T. Rasing, JETP Lett. 80, 293 (2004).

${ }^{2}$ A. G. Gavriliuk, S. A. Kharlamova, I. S. Lyubutin, I. A. Troyan, S. G. Ovchinnikov, A. M. Potseluiko, M. I. Eremets, and R. Boehler, JETP Lett. 80, 426 (2004).

${ }^{3}$ J.-C. Joubert, W. B. White, and R. Roy, J. Appl. Crystallogr. 1, 318 (1968)

${ }^{4}$ J. A. Campa, C. Cascales, E. Gutierrez-Puebla, M. A. Monge, I. Rasines, and C. Ruiz-Valero, Chem. Mater. 9, 237 (1997).

${ }^{5}$ S. Klimin, D. Fausti, A. Meetsma, L. N. Bezmaternykh, P. H. M. van Loosdrecht, and T. T. M. Palstra, cond-mat/0502423 (unpublished).
${ }^{6}$ A. I. Pankrats, G. A. Petrakovskii, L. N. Bezmaternykh, and O. A. Bayukov, JETP Lett. 99, 766 (2004).

${ }^{7}$ B. Lorenz, Y. Q. Wang, Y. Y. Sun, and C. W. Chu, Phys. Rev. B 70, 212412 (2004).

${ }^{8}$ F. Yen, C. R. dela Cruz, B. Lorenz, Y. Y. Sun, Y. Q. Wang, M. M. Gospodinov, and C. W. Chu, Phys. Rev. B 71, 180407(R) (2005).

${ }^{9}$ A. D. Balaev, L. N. Bezmaternykh, I. A. Gudim, V. L. Temerov, S. G. Ovchinnikov, and S. A. Kharlamova, J. Magn. Magn. Mater. 258-259, 532 (2003).

${ }^{10}$ M. Baran, H. Szymczak, S. A. Klimin, M. N. Popova, R. Z. Levitin, and B. V. Mill, JETP Lett. 84, 175 (1997). 
${ }^{11}$ C. dela Cruz, F. Yen, B. Lorenz, Y. Q. Wang, Y. Y. Sun, M. M. Gospodinov, and C. W. Chu, Phys. Rev. B 71, 060407(R) (2005).

${ }^{12}$ A. K. Zvezdin, S. S. Krotov, A. M. Kadomtseva, G. P. Vorob'ev, Y. F. Popov, A. P. Pyatakov, L. N. Bezmaternykh, and E. A. Popova, JETP Lett. 81, 335 (2005).

${ }^{13}$ K. P. Belov, A. K. Zvezdin, A. M. Kadomtseva, and R. Z. Levitin, Reorientational Transitions in Rare-Earth Magnets (Nauka, Moscow, 1980).

${ }^{14}$ Y. Hinatsu, Y. Doi, K. Ito, M. Wakeshima, and A. Alemi, J. Solid State Chem. 172, 238 (2003).

${ }^{15}$ R. Sachidanandam, T. Yildirim, A. B. Harris, A. Aharony, and O. Entin-Wohlman, Phys. Rev. B 56, 260 (1997).

${ }^{16}$ A. Muñoz, M. T. Casais, J. A. Alonso, M. J. Martinez-Lope, J. L.
Martinez, and M. T. Fernandez-Diaz, Inorg. Chem. 40, 1020 (2001).

${ }^{17}$ T. Lonkai, D. Hohlwein, J. Ihringer, and W. Prandl, Appl. Phys. A: Mater. Sci. Process. 74, S843 (2002).

${ }^{18}$ D. Petitgrand, S. V. Maleyev, P. Bourges, and A. S. Ivanov, Phys. Rev. B 59, 1079 (1999).

${ }^{19}$ M. Fiebig, C. Degenhardt, and R. V. Pisarev, J. Appl. Phys. 91, 8867 (2002).

${ }^{20}$ B. Lorenz, A. P. Litvinchuk, M. M. Gospodinov, and C. W. Chu, Phys. Rev. Lett. 92, 087204 (2004).

${ }^{21}$ R. Z. Levitin, E. A. Popova, R. M. Chtsherbov, A. N. Vasiliev, M. N. Popova, E. P. Chukalina, S. A. Klimin, P. H. M. V. Loosdrecht, D. Fausti, and L. N. Bezmaternykh, JETP Lett. 79, 423 (2004). 